\title{
A STUDY OF DEMOGRAPHY AND INDUCTION OUTCOME OF PEDIATRIC ACUTE LYMPHOBLASTIC LEUKEMIA IN A NEWLY-ESTABLISHED, RESOURCE-LIMITED SETTING IN INDIA.
}

\author{
Abhilasha Sampagar ${ }^{1}$, Neha Patil ${ }^{2}$, Merle Dias², Vishaka Kothiwale ${ }^{2}$, Nalla Anuraag \\ Reddy $^{2}$, Rashmi Hundekar ${ }^{2}$, and Priyadarshini Chougula ${ }^{2}$ \\ ${ }^{1} \mathrm{JNMC}$ \\ ${ }^{2}$ Jawaharlal Nehru Medical College
}

October 9, 2020

\begin{abstract}
BACKGROUND: Acute lymphoblastic leukemia(ALL) has survival rates of greater than $90 \%$ in developed nations. However various sociodemographic factors adversely affect outcome rates in low and middle income countries(LMIC). OBJECTIVE: To study induction outcome of ALL and various factors affecting it. METHODS: This was a prospective cohort study which enrolled 73 children up to age of 18 years with newly diagnosed ALL registered in our newly-established pediatric oncology division over duration of 2.5 years. Sociodemographic and clinical data was collected. Outcome was assessed using morphological remission, minimal residual disease(MRD) and mortality rate. RESULTS: Of the142 children with malignancies registered, 73 were ALL. Mean age was $7.09 \pm 4.07$ years, male to female ratio 1.15:1. 15(44.12\%) had severe acute malnutrition (SAM) and $14(41.17 \%)$ had moderate acute malnutrition (MAM). 27(69.23\%) children were undernourished. 62(84.93\%) were B-ALL, 11(15.07\%) TALL. $28.77 \%$ had WBC counts greater than 50X109/L. t(12;21) was the most common cytogenetic abnormality. $65.76 \%$ of the patients belonged to lower socioeconomic status. There were 10 dropouts. 59(93.65\%) patients completed induction of which $100 \%$ attained morphological remission and 54(91.53\%) were MRD negative. There were 4 mortalities, 2(50\%) due to sepsis and $2(50 \%)$ due to hemophagocytic lymphohistiocytosis(HLH). 41(65.08\%) children had morbidities during induction, febrile neutropenia being the commonest. CONCLUSIONS: Successful induction outcome rates at par with high income countries(HIC) can be achieved even in resource-limited settings of LMIC with support from government schemes and NGOs. Decentralized cancer care centres can effectively pave the way in reducing cancer mortality in children of lower socioeconomic status residing in rural areas.
\end{abstract}

\section{INTRODUCTION}

ALL is the most common childhood cancer. Considered invariably fatal until the 1960s, this disease has a 5-year survival rate of more than $90 \%$ in high income countries(HIC) today [1,2]. Diagnostic and treatment modalities for ALL have seen tremendous advancements over the past few decades. Despite of a higher incidence and constant rise in pediatric cancers in the developed countries, their success stories are ample[3]. With survival rates in developed countries approaching 100\%, there is a shift of research focus towards improvement of quality of life, reduction of morbidities and drug toxicities, targeted drug therapies and treatment of drug resistant leukemia[4]. On the other hand, LMIC like India with resource limited settings still struggle for optimal results in cancer care. A large proportion of treatment options for ALL is stationed in the major cities of the country. This reduces the accessibility and feasibility of adequate treatment for leukemic children in the peripheries. Since more than $65 \%$ of the Indian population resides in villages, access 
to cancer treatment is grossly hindered. Thus, pediatric cancer cases eventually approach adult oncologists for treatment resulting in compromised outcome. Poor socioeconomic conditions, cultural barriers, gender discrimination, malnutrition, delayed diagnosis and referral form some of the important yet modifiable factors attributed to poor outcomes in developing countries. Factors like age, presenting WBC counts, cytogenetics, pretreatment with steroids, extramedullary disease are seen to have an adverse effect on the treatment outcome[5].Mortality due to infections and treatment related toxicities, especially during the early phases of treatment are also of particular concern.

Induction forms the prime stage of treatment protocol for ALL. It is also the most sensitive phase associated with a high mortality and morbidity[2]. Delivery of intensive induction chemotherapy for ALL requires trained manpower and infrastructure which is usually available in established pediatric oncology centers in the urban areas in India. Challenges for delivering chemotherapy for newly- established pediatric oncology center in semi-urban areas are manifold. However, despite of resource-limited settings and a wide range of factors adversely influencing the induction outcome in ALL patients, a remission rate of more than $90 \%$ is an achievable target.

We started pediatric oncology center in September 2017 at a medical college attached hospital in North Karnataka. Aims of this prospective observational study were to determine demographic and clinical features and morbidity and mortality associated with induction chemotherapy for children with ALL.

\section{MATERIAL AND METHODS:}

\section{STUDY DESIGN : Prospective cohort study}

STUDY SETTING : This study was conducted in the Pediatric Oncology division of Department of Pediatrics of KLES Prabhakar Kore Charitable Hospital, Belagavi, Karnataka.

PARTICIPANTS : Children up to the age of 18 years with newly diagnosed ALL were enrolled consecutively between September 2017 and June2020. All patients received treatment based on standard arm of ICICLE (Indian Childhood Collaborative Leukemia Group) protocol. Ethical clearance was obtained. Informed consent was taken and confidentiality of data was maintained. Standard case report forms, specifically designed for this study were used to collect data which included demographic, clinical, laboratory features of all patients at the time of presentation. Factors leading to delayed diagnosis were also documented in a standardised format. Nutritional status was classified as per WHO and socioeconomic status as per modified BG Prasad classification. Participants were classified into standard risk and high risk according to NCI classification using age and WBC count. Standard risk was defined as WBC count $<50 \times 10^{9} / \mathrm{L}$ and age one to 9.99 years, while high risk was defined as $\mathrm{WBC}$ count [?]50x10 $/ \mathrm{L}$ at any age or age [?]10 years with any WBC count at presentation. A follow up case report form was filled for each patient at the end of induction phase which recorded morbidities during induction and induction outcome. The bacterial profile and antibiotic sensitivity pattern was studied in detail in order to understand the impact of infection on induction outcome.

\section{Diagnosis of Leukemia}

ALL was diagnosed when $20 \%$ lymphoblasts or more were present in the bone marrow (BM) aspirate/trephine biopsy. Flowcytometry was done for the immunophenotypic classification into B -cell ALL and T -cell ALL. Cytogenetic studies for common translocations including t $(12 ; 21), \mathrm{t}(9 ; 22), \mathrm{t}(4 ; 11)$ and $\mathrm{t}(1 ; 19)$ were tested by RT-PCR. Cytogenetics was done in all the patients except those who refused treatment. However, ploidy could be done in only 36 patients due to financial issues. Hyperdiploidy was defined as $>50$ chromosomes and hypodiploidy $<44$ chromosomes. Diagnostic lumbar puncture was done to determine CNS involvement \& patients were classified as CNS1, 2 and 3 categories based on standard criteria. Other investigations at the time of diagnosis included LFT, RFT, LDH levels, chest X-ray for mediastinal mass, abdominal ultrasound, 2D echocardiography and serology for HIV, HBsAg, HCV.

Prednisolone Response was defined as the cytoreduction (number of blasts per micro lit on day 8 of induction) to a 7 day prednisolone prephase. Patients were labelled as prednisolone good responder (PGR) 
and prednisolone poor responder (PPR) if they had $<1000$ blasts / $\mu \mathrm{L}$ in peripheral blood and [?]1000 blasts/ $\mu \mathrm{L}$ in peripheral blood after 1 week of steroid prephase respectively.

Minimal Residual Disease (MRD) was estimated by flow cytometry at the end of induction, and those who had MRD $<0.01 \%$ were considered MRD negative

\section{Risk stratification for treatment}

Risk stratification for ALL was done based on Children Oncology Group (COG) criteria adopted from National Cancer Institute (NCI) as mentioned in Table 1.

Table 1 ALL risk stratification for treatment purpose.

\section{TREATMENT}

In B-cell ALL, standard risk patients received 3 drugs in induction consisting of Prednisolone, vincristine and L-asparginase. Intermediate risk patients received 4 drugs during induction which also included 2 doses of daunorubicin. High risk patients received 4 drugs with 4 doses of daunorubicin. All T-cell ALL patients received 4 drugs in induction. T-cell ALL patients received dexamethasone after prednisolone prephase during induction. All the patients received 3 doses of intrathecal methotrexate. The duration of induction chemotherapy was 35 days.

\section{OUTCOMES}

Evaluation of the patient's therapeutic response was based on day 8 prednisolone response, morphological findings in BM and MRD on day 35 of induction. Patients were labeled to be in morphological remission if there were less than $5 \%$ blasts in the marrow with normal trilineage hematopoiesis and MRD negative if values were $<0.01 \%$.

\section{SAMPLE SIZE}

A total of 73 patients were enrolled.

\section{STATISTICAL ANALYSIS}

Data was analysed using R software version 3.6.1 and Excel. Categorical variables were given in the form of frequency table. Categorical variables were compared using chi-square test. Cochran Armitage test was used to study the linear trend in prevalence of binary outcome of interest over ordinal data. In case of any violation of assumptions of chi-square test, simulation was done for chi square test.

\section{RESULTS:}

Over a duration of 2.5 years, a total of 142 children between the age of 0-18yrs, with different malignancies presented to our centre (Table2). ALL(73) was the most common (51.4\%) malignancy.

\section{Table 2 Distribution of total number of pediatric oncological cases}

\section{Demographic and clinical characteristics of ALL cases}

The mean age was 7.09 \pm 4.07 years (range 0.8-17.2yrs) and male to female ratio was 1.15:1. Demographic, nutritional, socioeconomic and clinical features are illustrated in Table 3.

The mean duration from the onset of symptoms to referral to our oncology centre was 42.7 days. Fever was the most common presenting symptom in $27(36.5 \%)$ children. $37(50.7 \%)$ cases referred to us had an initial diagnosis of leukemia. However, in the rest $36(49.3 \%)$ of cases, varied diagnosis like juvenile idiopathic arthritis in 8(10.96\%); megaloblastic anemia in 5(6.85\%) and aplastic anemia in 1 (1.37\%)due to pancytopenia; tubercular lymphadenitis in $4(5.48 \%)$; dengue hemorrhagic fever in $7(9.59 \%)$, brucellosis in $2(2.74 \%)$, typhoid fever in $3(4.11 \%)$, pyrexia of unknown origin in $2(2.74 \%)$; and pneumonia with effusion in $3(4.11 \%)$ due to mediastinal mass formed factors leading to delayed referral. $7(9.59 \%)$ cases were pretreated with steroids. The mean duration of steroid administration was 33.75 days. 
Table 3 Table showing Sociodemographic factors and clinical features of the study participants and association with risk of mortality or morbidity during induction.

As per immunophenotype, 62(84.93\%) cases were B-cell ALL and 11(15.07\%) were T-cell ALL. There were10 dropouts of which 9 refused and 1 abandoned treatment. The reasons for refusal and abandonment are mentioned in Table 4. Cytogenetics could not be done for drop out cases. Out of 63 subjects, $\mathrm{t}(12 ; 21)$ was seen in $11(17.46 \%)$ cases, $\mathrm{t}(1 ; 19)$ in $8(12.7 \%)$ cases, $\mathrm{t}(9 ; 22)$ in $3(4.76 \%)$ and MLL was positive in 1. Ploidy was done for $36(49.3 \%)$ patients, of which $9(25 \%)$ were hypodiploid, $8(22 \%)$ were hyperdiploid and $19(52.8 \%)$ were aneuploid cases. Risk stratification as per NCI risk criteria and ICICLE protocol showed high risk predominance (Table 3,4 ).

\section{Table 4 Outcome of induction chemotherapy}

\section{Treatment outcome:}

The mean duration of induction chemotherapy was 37.2 days. Of the total 63 patients who underwent treatment, 59(93.7\%) could complete the induction chemotherapy and all of them went into morphological remission. A total of 5(8.5\%) patients were MRD positive at the end of induction of which 4 belonged to high risk and 1 to standard risk category. Of the 4 (6.3\%) deaths during induction, 2 were due to infections with multidrug resistant organisms (Acinetobacter \& klebsiella) and two were due to infection associated HLH. 3 out of 4 mortality cases had severe acute malnutrition (SAM). The morbidities during induction chemotherapy are shown in the Table 4 . Infection was the commonest complication.

There were total 37(58.7\%) episodes of febrile neutropenia. Blood culture was sent during each episode of febrile neutropenia. A total of 21(56.8\%) cultures were positive of which $13(62 \%)$ cultures grew gram negative and $8(38 \%)$ grew gram positive bacteria. Out of gram-negative bacterial infections $4(30.8 \%)$ were klebsiella, 2(15.4\%) were pseudomonas, 3(23.1\%) were Acinetobacter , 2(15.4\%) Ecoli and 2(15.4\% )burkholderia. Among the gram positive bacteria 5(62.5\%) were methicillin resistant staph aureus, $2(25 \%)$ were streptococci and $1(12.5 \%)$ was enterococci. Multi drug resistant strains were seen in $6(28.6 \%)$ patients out of which $5(83.3 \%)$ were gram negative and $1(16.6 \%)$ was gram positive. 2 cultures grew candida along with other bacteria. One patient had parvovirus infection. Another interesting finding of our study was that, all the patients who harboured MDR bugs were pre-treated with multiple broad-spectrum antibiotics before being referred to our Centre as the diagnosis was delayed in the cases and they were labelled as cases of pyrexia of unknown origin.

\section{Analysis of risk factors for mortality or morbidity:}

We analysed five factors using Chi-square test and Cochrane Armitage trend test whether they predict increased risk of mortality or morbidity during induction chemotherapy- age, gender, socioeconomic status, nutritional status, risk group. None of the factors showed statistically significant association. (Table 3)

\section{DISCUSSION:}

The outcome of children with ALL is suboptimal in LMIC compared to HIC, toxicity during induction being one of the important factors responsible for it. This prospective study primarily aimed at determining the outcome of induction therapy in the management of ALL in a newly -established, resource-limited setting in a sub-urban area of India.

Of the 142 cancer cases registered at our 2.5 year-old pediatric oncology center, more than half(51.41\%) were ALL. The mean age of presentation of 7.9 years was slightly higher than those reported in Western countries (2-3 years) but comparable with the median age of 5 to 10 years seen in a comprehensive study in India[7, 8].

Only a slight male preponderance (53.42\%) was observed in our study. The male to female ratio of 1.15:1 seen in our study was much lesser than gender ratios reported from many Indian hospital based and population based studies[8]. Gender bias and neglect towards the female child were seen often during the initial 6 months 
of opening up of our center and were majorly resolved with the provision of Government schemes and NGO support for finance. Thereafter, we were fortunate to not have witnessed a single gender based refusal.

The nutrition of a child plays an important role in the course of disease and its outcome. In our study, the incidence of malnutrition was exceedingly high, $85.3 \%$ below 5 years and $69.23 \%$ above 5 years of age) compared to study from Bangladesh (53.03\%) and West Bengal (72.19\%) [9,10]. A study from Lucknow, India highlighted pretreatment hypoalbuminemia, vitamin B12 and folate deficiencies to be associated with a higher mortality due to drug toxicities during induction[11]. Although baseline vitamin B12 and folate levels could not be estimated in our study, 19(26.03\%) children who had hypoalbuminemia, faced difficult induction. Almost all children with SAM witnessed toxicity during induction. Malnutrition was more common in children of rural settings and those with a delayed diagnosis. Best attempts were made to correct malnutrition with the help of a trained nutritionist and high protein-energy diet individualized for each patient.

Two third $(65.76 \%)$ of the patients belonged to lower socioeconomic status (Class IV and V as per modified B. G. Prasad classification), of which $37(77 \%)$ belonged to rural areas of North Karnataka. Treatment of leukemia is lengthy and demanding. It requires prolonged hospital stay with parents having to sacrifice their jobs for adequate treatment and care of the child. Hence, parents from low economic backgrounds often tend to refuse or abandon treatment midway due to exhaustion of monetary resources. Parents also find it hard to relocate to major cities where majority of cancer care is concentrated. In our study, financial issue was the chief reason for refusal/ abandonment of treatment. Parental education was also a determining factor in overall nutrition and hygiene of a child. Negligence and myths that cancer is an incurable disease also affected the outlook of many parents towards acceptance of chemotherapy. Educating the parents and providing psychological support became important in this setting.

We observed mean duration 42.7 days from initial symptoms to referral to our center. Almost half (49.3\%) of children referred to us had an alternative initial diagnosis contributing to delay in diagnosis of ALL. Moreover, this led to undesirable therapy which can affect the outcome of ALL. For example, 7 out of 8 patients initially diagnosed as JIA received treatment with steroids. Also, patients diagnosed as PUO were treated with multiple higher antibiotics. A study on pattern of mortality in childhood ALL in North India revealed a higher symptom diagnosis interval ( $53.9 \pm 8.3$ days) in mortality cases compared to $39.6 \pm 7.9$ days in survivors[12].

$28.77 \%$ of the cases presented with WBC counts higher than $50 \times 10^{9} / \mathrm{L}$ of which more than half $(57.14 \%)$ had counts greater than $100 \mathrm{X} 10^{9} / \mathrm{L}$. These counts were significantly higher when compared to studies in the west but in concordance with studies from LMIC[8,13]. As per the NCI classification, three-fifth $(60.27 \%)$ of patients presenting to us were high risk. This reflected delayed referral, higher tumor burden and a potential adverse prognosis at the time of presentation. Leukopenia, coupled with nonspecific and constitutional symptoms of ALL can further confound its diagnosis. In our study, of the 11(15.07\%) cases presenting with leucopenia $9(81.81 \%)$ were initially diagnosed differently and treated for varied causes.

T-cell ALL constituted $15.07 \%$ of the total cases which corresponded to its usual incidence[14]. More than one fourth $(27.27 \%)$ of cases with T-ALL had CNS 3 disease. Also, 3 out of 4(75\%) cases with CNS involvement were of the T-cell subtype.100\% of the T-ALL cases presented with mediastinal masses mimicking pneumonias. This incidence was grossly higher compared to a multi-institutional study in Pakistan (34.8\%)[15]. Moreover, higher incidence of tuberculosis in developing countries like India can lead to incorrect diagnosis of the disease. In our study, 4 (5.48\%) of the total cases and $3(27.27 \%)$ of T-ALL cases were initially diagnosed as tubercular lymphadenitis and treated for the same.

Incidence of t(12:21) (17.46\%) in our cohort was slightly higher than studies from India(9.4-13.7\%) however incidence of Philadelphia chromosome $(4.76 \%)$ was much lesser than its known incidence in childhood ALL(12\%) [8,16 ]. A higher incidence of hypodiploidy (25\%) was observed in our study[17]. Hypodiploidy, being a poor prognostic indicator can lead to increased relapses and treatment failure even in advanced settings.

Our induction mortality rate of $6.3 \%$ is higher than that seen in HIC[18]. Review of Indian data in ALL by 
RS Arora et al covering 3761 children reported a death rate of 2-13\% during induction[8].In our study, all deaths were due to bacterial infection and its complications. Bacterial sepsis is indeed the leading cause of mortality during induction[6]. Amongst various morbidities observed in our study, infection especially due to gram negative bacteria was most common as also seen in a South Indian study[19]. We could not find any statistically significant factor predicting induction mortality or morbidity probably due to smaller sample size.

Induction outcome based on morphological and MRD assessment showed promising results. Morphological remission was seen in $100 \%$ of the cases who completed the induction chemotherapy. Minimal residual disease (MRD) is currently the most powerful prognostic indicator in ALL and a negative rate of $91.52 \%$ at the end of induction reflects the success of treatment at par with established centers[8]. The day 8 prednisolone response was poor in $7(9.72 \%)$ of the cases who initiated treatment(72) of which $2(28.57 \%)$ were MRD positive.

In conclusion, high tumor load, low socioeconomic status, malnutrition, delayed diagnosis and infections confer substantial challenges during induction chemotherapy in children with ALL in sub -urban pediatric oncology centers. Nevertheless, fairly successful outcomes can be achieved with the help of trained personnel, multidisciplinary team and financial support from government schemes and NGOs. Hence, decentralized cancer care centres can effectively pave the way in reducing paediatric cancer mortality in children of lower socioeconomic status residing in rural areas. Furthermore, it will lead to expansion of treatment options to rural settings which form residence to more than $65 \%$ of the Indian population, thus fighting the major hurdles in access to cancer care. Also, increasing awareness regarding early signs and symptoms of ALL can help in early identification of the disease and prevent the child from advancing into higher risks of mortality.

The authors declare that there is no conflict of interest.

\section{ACKNOWLEDGEMENTS:}

We thank CANKIDS, LEUKEMIA CRUSADERS and CUDDLES FOUNDATION for financial and nutritional support to the children.

We thank Dr. N. S. Mahantashetti, Principal, JNMC Belagavi for her support in establishing the pediatric oncology unit.

We also thank Dr. Vibha Bafna and Dr. Sandip Bartakke for training, encouragement and motivation.

\section{REFERENCES}

1. Pui CH, Evans WE. A 50-year journey to cure childhood acute lymphoblastic leukemia. InSeminars in hematology 2013 Jul 1 (Vol. 50, No. 3, pp. 185-196). WB Saunders.

2. Cools J. Improvements in the survival of children and adolescents with acute lymphoblastic leukemia. Haematologica. 2012 May;97(5):635.

3. Hubbard AK, Spector LG, Fortuna G, Marcotte EL, Poynter JN. Trends in international incidence of pediatric cancers in children under 5 years of age: 1988-2012. JNCI cancer spectrum. 2019 Mar;3(1):pkz007.

4.Pui CH, Yang JJ, Bhakta N, Rodriguez-Galindo C. Global efforts toward the cure of childhood acute lymphoblastic leukaemia. The Lancet Child \& Adolescent Health. 2018 Jun 1;2(6):440-54.

5. Lee JW, Cho B. Prognostic factors and treatment of pediatric acute lymphoblastic leukemia. Korean journal of pediatrics. 2017 May;60(5):129.

6.Kiem Hao T, Nhu Hiep P, Kim Hoa NT, Van Ha C. Causes of Death in Childhood Acute Lymphoblastic Leukemia at Hue Central Hospital for 10 Years (2008-2018). Global Pediatric Health. 2020 Jan;7:2333794X20901930.

7. Howlader N, Noone AM, Krapcho M, Garshell J, Neyman N, Altekruse SF, Kosary CL, Yu M, Ruhl J, Tatalovich Z, Cho H. SEER cancer statistics review, 1975-2010. Bethesda, MD: National Cancer Institute. 
2013 Apr 21;21:12.

8. Arora RS, Arora B. Acute leukemia in children: A review of the current Indian data. South Asian journal of cancer. $2016 \mathrm{Jul} ; 5(3): 155$.

9. Hafiz MG, Mannan MA. Lymphoblastic Leukemia and Its Effect on Induction of Remission. Mymensingh Med J. 2008 Jul;17(2 Suppl).

10. Roy A, Saha A, Chakraborty S, Chattopadhyay S, Sur PK. Effects of pre-existing undernutrition on treatment-related complications and treatment outcomes in children with acute lymphoblastic leukemia: A tertiary care center experience. Clinical Cancer Investigation Journal. 2013 Apr 1;2(2):143.

11.Tandon S, Moulik NR, Kumar A, Mahdi AA, Kumar A. Effect of pre-treatment nutritional status, folate and vitamin B12 levels on induction chemotherapy in children with acute lymphoblastic leukemia. Indian pediatrics. 2015 May 1;52(5):385-9.

12. Marwaha RK, Kulkarni KP, Bansal D, Trehan A. Pattern of mortality in childhood acute lymphoblastic leukemia: experience from a single center in northern India. Journal of Pediatric Hematology/Oncology. 2010 Jul 1;32(5):366-9.

13.Jaime-Perez JC, Garcia-Arellano G, Herrera-Garza JL, Marfil-Rivera LJ, Gomez-Almaguer D. Revisiting the complete blood count and clinical findings at diagnosis of childhood acute lymphoblastic leukemia: 10year experience at a single center. Hematology, transfusion and cell therapy. 2019 Mar;41(1):57-61.

14. Chiaretti S, Foa R. T-cell acute lymphoblastic leukemia. Haematologica. 2009 Feb;94(2):160-2.

15. Fadoo Z, Nisar I, Yousuf F, Lakhani LS, Ashraf S, Imam U, Zaheer J, Naqvi A, Belgaumi A. Clinical features and induction outcome of childhood acute lymphoblastic leukemia in a lower/middle income population: A multi-institutional report from Pakistan. Pediatric blood \& cancer. 2015 Oct;62(10):1700-8.

16. Pui CH, Roberts KG, Yang JJ, Mullighan CG. Philadelphia chromosome-like acute lymphoblastic leukemia. Clinical Lymphoma Myeloma and Leukemia. 2017 Aug 1;17(8):464-70.

17. Guru FR, Muzamil J, Bashir S. Acute lymphoblastic leukemia, the Indian scenario. MOJ Cell Sci Rep. 2018;5(2):33-7.

18. Seif AE, Fisher BT, Li Y, Torp K, Rheam DP, Huang YS, Harris T, Shah A, Hall M, Fieldston ES, Kavcic M. Patient and hospital factors associated with induction mortality in acute lymphoblastic leukemia. Pediatric blood \& cancer. 2014 May;61(5):846-52.

19. Rajeswari B, Nair RK, Guruprasad CS, Nair M, Thankamony P, Parukutty K. Infections during induction chemotherapy in children with acute lymphoblastic leukemia-Profile and outcomes: Experience from a cancer center in South India. Indian Journal of Medical and Paediatric Oncology. 2018 Apr 1;39(2):188.

Legends:

Table 1 ALL risk stratification for treatment purpose.

Table 2 Distribution of total number of pediatric oncological cases

Table 3 Table showing Sociodemographic factors and clinical features of the study participants and association with risk of mortality or morbidity during induction.

Table 4 Outcome of induction chemotherapy

\section{Hosted file}

PCB TABLE 1.pdf available at https://authorea.com/users/365715/articles/485709-a-study-ofdemography-and-induction-outcome-of-pediatric-acute-lymphoblastic-leukemia-in-a-newlyestablished-resource-limited-setting-in-india 


\section{Hosted file}

PBC table 2.pdf available at https://authorea.com/users/365715/articles/485709-a-study-ofdemography-and-induction-outcome-of-pediatric-acute-lymphoblastic-leukemia-in-a-newlyestablished-resource-limited-setting-in-india

\section{Hosted file}

PBC table 3.pdf available at https://authorea.com/users/365715/articles/485709-a-study-ofdemography-and-induction-outcome-of-pediatric-acute-lymphoblastic-leukemia-in-a-newlyestablished-resource-limited-setting-in-india

\section{Hosted file}

PBC table 4.pdf available at https://authorea.com/users/365715/articles/485709-a-study-ofdemography-and-induction-outcome-of-pediatric-acute-lymphoblastic-leukemia-in-a-newlyestablished-resource-limited-setting-in-india 